\title{
An Exposition of Polygonal Approximation of Circle
}

\section{PALASH KHANRA}

\begin{abstract}
In this article, it is attempted to discuss how do we can create an infinite number of circles from a single circle, using its tangents with a pattern $\left(P_{X} ; \mathrm{X} \geq 3\right)$ and what is the process to reach up into a single point from a given circle. The time to get a new circle from its predecessor circle can be reduced just by changing the pattern. The more we repeat the polygonal pattern the better we can observe that the set of all end intersecting points of the tangents becoming similar ${ }^{1}$ to the set ${ }^{2} \mathrm{R}$. The pattern behind both the radius of the successor circles and predecessor circles is also discussed. Most interestingly, when we apply the $P_{\infty}$ pattern of tangents on the single circle, then all the infinitely many successor circles merge into a single one, almost without taking any time and it takes infinite time to reach up into a single point from the given circle, for that pattern. In the whole process, polygonal approximation and sequences play a vital role.
\end{abstract}

2010 Mathematics Subject Classification • 00A05, 00A08, 97G99

Keywords and phrases . Circle generation; n-Regular polygons; Approximations; Sequential-patterns

\section{INTRODUCTION.}

Polygons, along with lines and circles, constitute the earliest collection of Geometric figures studied by humans [1]. By polygon, we mean a closed figure with a finite number $n$ of sides ( $\mathrm{n}$ vertices)( $\mathrm{n} \geq 3)$ each of which is a line segment. The polygonal approximation of circles is commonly known throughout the Mathematics world. In polygonal approximation, we are interested in lengths and these lengths are approximations to the arc length of the curve. Increasing the value of the number of sub-intervals into which domain is divided, increases the accuracy of the approximation.

\footnotetext{
${ }^{1}$ Similar means they have the same potency. Cutting the circumference of a circle and stretching it, gives us an interval on the real axis. Now any non-empty non-singleton interval and $\mathrm{R}$ have the same potential. This equipotency in between an arbitrary non-empty non-singleton interval and $\mathrm{R}$ can be easily checked using projection

${ }^{2} \mathrm{R}$ means, the set of real numbers.
} 
The first work of circle approximation dates back to Archimedes of Syracuse (287212BC), one of the greatest mathematicians of the ancient world. Archimedes approximated the area of a circle by using the Pythagorean theorem to find the areas of two regular polygons: the polygon inscribed within the circle and the polygon within which the circle was circumscribed [4]. Later many mathematicians worked on this. Some of them use parametric polynomial curves and Quadratic Bézier curves to approximate circular arcs. L Yong-Kui has worked on circle approximation and its generation (1993). He introduces a new algorithm for the generation of the circle, using the intersecting polygon instead of the inscribed polygon, which greatly reduces the error [5]. In recent years 2011, Józef Borkowski had done work on 'Minimization of Maximum Errors In Universal Approximation of The Unit Circle By a Polygon' [6]. In 2019, J. M. Carnicer · E. Mainar · J. M. Peña constructs a six-dimensional space of polynomials containing approximations of trigonometric functions. In this way, they represented an approximation of a circle as a parametric curve in this space [7].

Researchers gave many algorithms and interesting ways to approximate circles. A very few worked on the generation of circles. Now some questions arise in our mind that, is it possible to create infinitely many successor and predecessor circles from a single circle, using its tangents? If yes! then how the time varies for getting new circles again and again ? what is the process to reach up into a single point from a given circle? What is the pattern behind the radius of the successor circles and distances between any two nearby successor circles? Also what for the predecessor circles? Though ongoing researches are more advanced but to investigate all those queries, we should look upon it as a child. Then we can resolve those things.

In this article, we will try to illustrate and resolve all those queries.

\section{PROCEDURE.}

Let us consider that, we have a single circle (Figure 1) and any tangents of the circle looks like (Figure 2). Here, we will deal with the regular polygonal pattern of tangents.

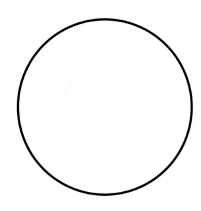

Figure 1: circle

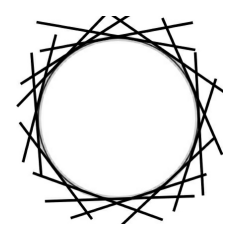

Figure 2: Circle with it's tangents 


\subsection{SUCCESSOR CIRCLES.}

\subsubsection{VISUALISATION TO THE PROCESS.}

Let's begin with the simplest regular polygonal pattern i.e. the triangular pattern of tangents. First we take three tangents with triangular pattern on the given single circle. Then we have Figure 3.

Define, $b_{i}$ : for all $\mathrm{i}$ belongs to $\mathrm{N}$, as end-intersecting points of tangents on the circle. It

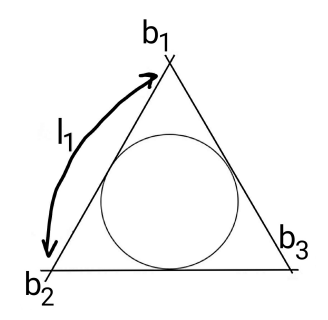

Figure 3: $n=1$

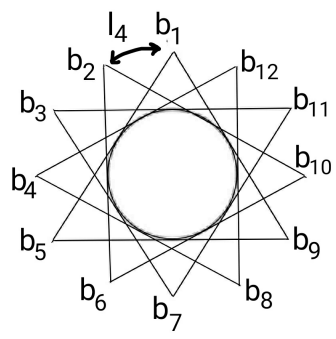

Figure 5: $n=4$

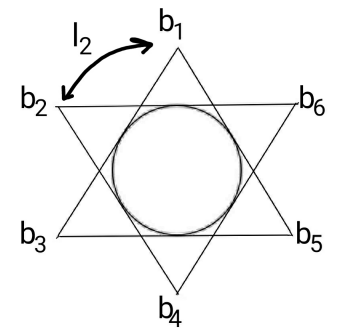

Figure 4: $n=2$

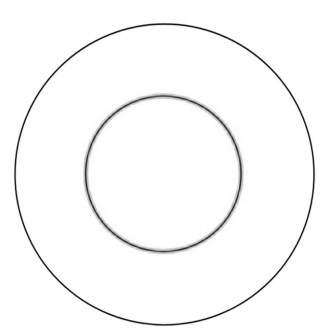

Figure 6: 1st successor circle, $n \longrightarrow \infty$

is assumed that all end-intersecting points are distributed in a precise way. Meaning, for each $\mathrm{n}=\mathrm{k}$ ( Here, $\mathrm{n}=\mathrm{k}$ meaning we are applying the mentioned polygon pattern $\mathrm{k}$ times ), $\mathrm{k}$ belongs to $\mathrm{N} ; d_{u}\left(b_{1}, b_{2}\right)=d_{u}\left(b_{2}, b_{3}\right)=\ldots=d_{u}\left(b_{i}, b_{i+1}\right)$; for all $\mathrm{i}$ belongs to $\mathrm{N}$.

Here, $d_{u}$ symbolises the Euclidean distance ${ }^{3}$. Define $d_{u}\left(b_{i}, b_{i+1}\right)=l_{k}$ for $\mathrm{n}=\mathrm{k}$.

The above geometric treatments shows that we have a decreasing sequence here, $\left(L_{n}\right)_{n=1}^{\infty}=\left\{l_{1}, l_{2}, l_{3}, \ldots, l_{n}, \ldots\right\}$ where, $l_{1}>l_{2}>l_{3}>\ldots>l_{n}>\ldots$ It can be easily checked that this sequence converges to 0 and then we will have our $1_{s t}$ successor circle and the set of all $b_{i}{ }^{\prime} s$ becoming similar to the set of real numbers. One can easily see from the figures that the sequence is bounded and monotonic decreasing and can understand how does, $d_{u}\left(b_{i}, b_{i+1}\right) \longrightarrow 0$, for all i belongs to $\mathrm{N}$, when $n \longrightarrow \infty$.

\footnotetext{
${ }^{3}$ In the whole article, we will use $d_{u}$ as a notation of Euclidean distance.
} 
Now $2_{n d}$ successor circle can be obtained applying the same idea of tangents pattern (here, triangular) on the $1_{s t}$ successor circle.

Let, $c_{i}$ : for all $\mathrm{i}$ belongs to $\mathrm{N}$, are the end intersecting points of tangents on the $1_{s t}$ successor circle. It is assumed that, those points are distributed in a precise way ,means for each $\mathrm{n}=\mathrm{k}$ : $\mathrm{k}$ belongs to $\mathrm{N} ; d_{u}\left(c_{1}, c_{2}\right)=d_{u}\left(c_{2}, c_{3}\right)=\ldots=d_{u}\left(c_{i}, c_{i+1}\right)$; for all $\mathrm{i}$ belongs to $\mathrm{N}$.

Define, for $\mathrm{n}=\mathrm{k}, d_{u}\left(c_{i}, c_{i+1}\right)=m_{k}$. Then a decreasing sequence can be obtained and

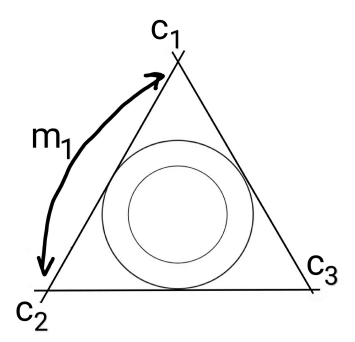

Figure 7: $n=1$

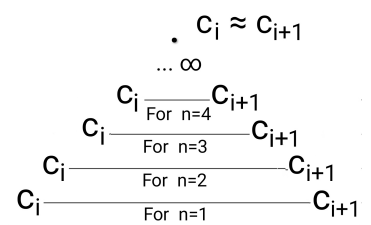

Figure 8: Varying lengths of $m_{i}^{\prime} s$

it is defined by, $\left(M_{n}\right)_{n=1}^{\infty}=\left\{m_{1}, m_{2}, m_{3}, \ldots, m_{n}, \ldots\right\}$. Now, $d_{u}\left(c_{i}, c_{i+1}\right) \longrightarrow 0$ when $n \longrightarrow \infty$; for all $\mathrm{i}$ belongs to $\mathrm{N}$ and it can be easily shown that, this sequence converges to 0 . Therefore, the Set of all, $c_{i}{ }^{\prime} s$ becoming similar to the set R. So, now we have our $2_{n d}$ successor circle.

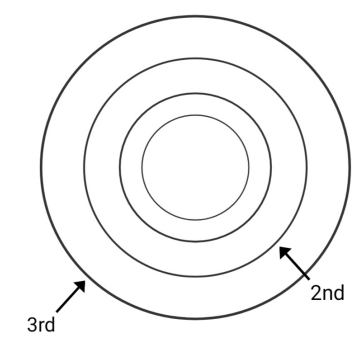

Figure 9: 2nd and 3rd successor circles

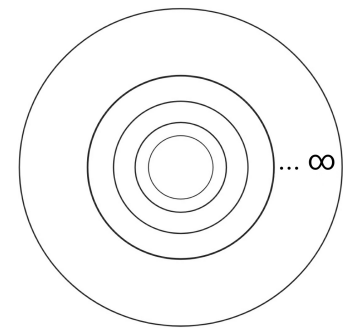

Figure 10: Infinitely many successor circles

Our $3_{r d}$ successor circle can be obtained, again applying the same procedure ( means, applying the mentioned polygonal pattern of tangents on the $2_{n d}$ successor circle, we will have a sequence of Euclidean distances of the end intersecting points of the tangents on $2_{n d}$ successor circle and when we apply that pattern for infinitely many times then that sequence converges to 0 ). In Fig. 8 we attempted to give an idea of how does the Euclidean distance between $c_{i}$ and $c_{i+1}$ i.e. the lengths of $m_{i}{ }^{\prime} s$ decrease when the value of $\mathrm{n}$ increases. That distance is going to be zero when $n \longrightarrow \infty$. Now, infinitely many 
successor circles can be obtained, repeating this sequential process infinitely many times.

Now, we will deal with the $2_{n d}$ simplest regular polygon i.e. square ( basically the square pattern of tangents on circles ). Here also it is assumed that, the end-intersecting points ( say $b_{i}{ }^{\prime} s$ ) of tangents are distributed in a precise way ( means for each $\mathrm{n}=\mathrm{k}: \mathrm{k}$ belongs to $\mathrm{N} ; d_{u}\left(b_{1}, b_{2}\right)=d_{u}\left(b_{2}, b_{3}\right)=\ldots=d_{u}\left(b_{i}, b_{i+1}\right)$; for all i belongs to $\left.\mathrm{N}\right)$.

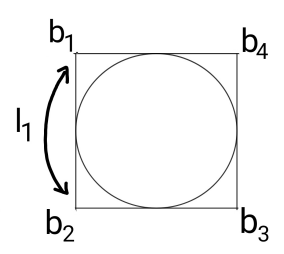

Figure 11: $n=1$

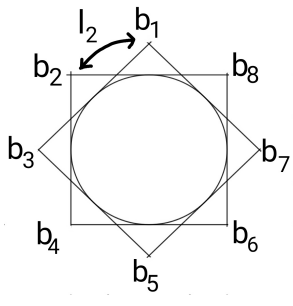

Figure 12: $n=2$

Define, $d_{u}\left(b_{i}, b_{i+1}\right)=l_{k}$, for $\mathrm{n}=\mathrm{k}: \mathrm{k}$ belongs to $\mathrm{N}$.

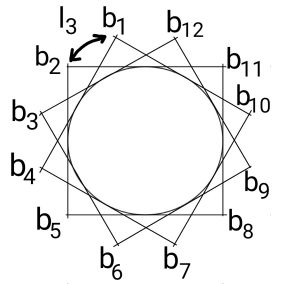

Figure 13: $n=3$

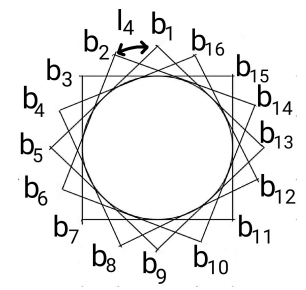

Figure 14: $n=4$

It can be easily checked that a decreasing sequence of $l_{k}{ }^{\prime} s,\left(L_{n}\right)_{n=1}^{\infty}=\left\{l_{1}, l_{2}, l_{3}, \ldots, l_{n}, \ldots\right\}$ can be obtained and converging to 0 and then we will have our $1_{s t}$ successor circle ( using a square pattern ) and the set of all $b_{i}{ }^{\prime} s$ becoming similar to the set $\mathrm{R}$.

Let, $c_{i}$ : for all $\mathrm{i}$ belongs to $\mathrm{N}$, are the end intersecting points of tangents on the $1_{s t}$ successor circle. It is assumed that, those points are distributed in a precise way ,means for each $\mathrm{n}=\mathrm{k}$ : $\mathrm{k}$ belongs to $\mathrm{N} ; d_{u}\left(c_{1}, c_{2}\right)=d_{u}\left(c_{2}, c_{3}\right)=\ldots=d_{u}\left(c_{i}, c_{i+1}\right)$; for all $\mathrm{i}$ belongs to $\mathrm{N}$.

Define, $d_{u}\left(c_{i}, c_{i+1}\right)=m_{k}$, for $\mathrm{n}=\mathrm{k}$; $\mathrm{k}$ belongs to $\mathrm{N}$.

Here also the sequence $\left(M_{n}\right)_{n=1}^{\infty}=\left\{m_{1}, m_{2}, m_{3}, \ldots, m_{n}, \ldots\right\}$ converges to 0 and the set of all $c_{i}{ }^{\prime} s$ becoming similar to the set of real numbers i.e. R. Then our $2_{n d}$ successor circle is obtained. Infinitely many successor circles can be obtained, repeating this sequential process infinitely many times. 


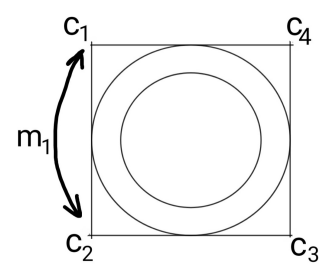

Figure 15: $\mathrm{n}=1$

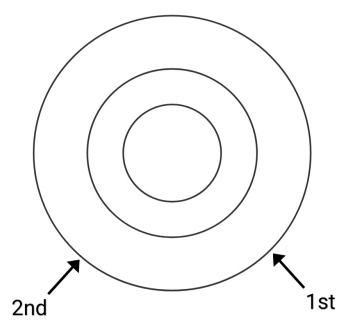

Figure 17: $1_{s t}$ and $2_{n d}$ successor circle

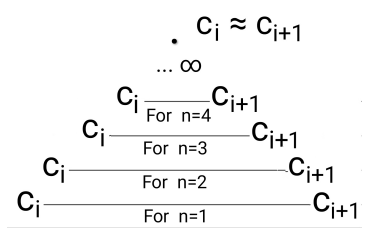

Figure 16: Varying length's of $m_{i}{ }^{\prime} s$

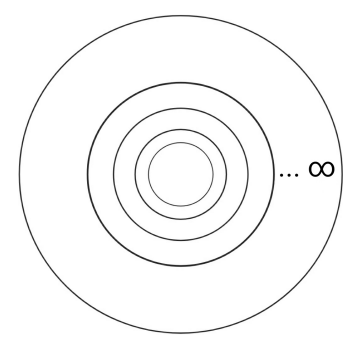

Figure 18: Infinitely many successor circles

In previous portions,the triangular pattern and square pattern are discussed. But the interesting fact is, infinite number of circles from a single circle using it's tangents, can be obtained with any polygonal pattern (applying sequential idea).

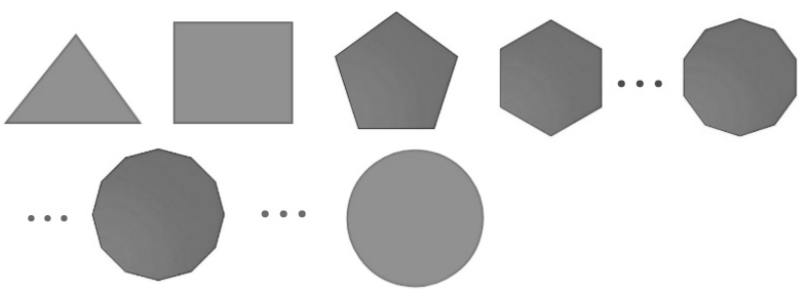

Figure 19: n-Regular Polygons

The above figure gives us an idea of the polygonal approximation of a circle. When the number of sides of successor polygons increases then the Euclidean distance between the vertices decreases and when the distance almost becomes 0 then the polygon almost transformed into a circle (Increasing the value of the number of sub-intervals into which, the domain is divided, increases the accuracy of approximation ). Here also a sequence of distances of vertices can be obtained and that sequence converging to 0 .

The algorithm behind the whole process of getting infinitely many successor circles from a given single circle is mentioned below. 


\subsubsection{ALGORITHM.}

Step 1 : Take a single circle.

Step 2: Take tangents on the single circle with a polygonal pattern ( one can take any polygonal pattern; $\mathrm{n} \geq 3$ ).

Step 3: Apply the same polygonal pattern again and again.

Step 4: For each $n=k$ ( $k$ belongs to $N$ ), the euclidean distance between any two nearby end-intersecting points of tangents should be equal. ( here, $\mathrm{n}=\mathrm{k}$ means we are applying the mentioned polygonal pattern for $\mathrm{k}$ times ).

Step 5: The Euclidean distance between any two successive end-intersecting points decreases when the value of $n$ increases.

Step 6: For $\mathrm{n}$ tending to infinity, that distance is going to be zero.

Step 7: Hence, the $1_{s t}$ successor circle is obtained.

Step 8 : Repeat the same procedure for $1_{s t}$ successor circle to get the $2_{n d}$ one. Repeat this process again and again for getting more and more successor circles.

\subsubsection{TIME.}

Let's denote the regular polygons by, $P_{X}(\mathrm{X} \geq 3)$, where $\mathrm{X}$ denotes the number of vertices.

Let, $T_{1}$ be the time to get the $1_{s t}$ successor circle from the given single circle using the $P_{3}$ pattern of tangents. Now, whenever we apply the $P_{3}$ pattern for the $1_{s t}$ time, then 3 end-intersecting points of the tangents of the single circle, are obtained. Applying $P_{3}$ pattern for the $2_{n d}$ time, we have 6 end-intersecting points. Continuing this process, we will have $9,12,15, \ldots$ so on. Observe that, a sequence arises here and it is defined by,

$$
\left(P_{3 n}\right)_{n=1}^{\infty}=\{3,6,9,12, \ldots, 3 n, \ldots\} \text { i.e. }(3 n)_{n=1}^{\infty}
$$

Now, $T_{1}$ depends on the rate of convergence of sequence $\left(\frac{1}{P_{3 n}}\right)_{n=1}^{\infty}$ i.e. how quickly the terms of the sequence converge to the limit zero.

Let, $T_{2}$ be the time to get the $1_{s t}$ successor circle using $P_{4}$ pattern of tangents. It can be easily checked that a sequence of end-intersecting points of tangents can be obtained and it is defined by,

$$
\left(P_{4 n}\right)_{n=1}^{\infty}=\{4,8,12,16, \ldots, 4 n, \ldots\} \text { i.e. }(4 n)_{n=1}^{\infty}
$$

Here, $T_{2}$ depends on the rate of convergence of sequence $\left(\frac{1}{P_{4 n}}\right)_{n=1}^{\infty}$ i.e how quickly the terms of the sequence converges to 0 .

Now proceeding in a similar way, it can be observed that, if $T_{K}: \mathrm{K}$ belongs to $\mathrm{N}$ , be the time to get the $1_{s t}$ successor circle from the single circle using $P_{K+2}$ pattern. 
Then, $T_{K}$ depends on the rate of convergence of sequence, $\left(\frac{1}{P_{(K+2) n}}\right)_{n=1}^{\infty}$. We need to compare all those sequences, on which $T_{K}{ }^{\prime} s$ depends. It can be easily checked that, for all $\mathrm{n}$ belongs to $\mathrm{N}, \frac{1}{3 n}>\frac{1}{4 n}>\frac{1}{5 n}>\ldots>\frac{1}{K n}>\ldots$ Here, any $\frac{1}{K n}$ defines the terms of sequence $\left(\frac{1}{K n}\right)_{n=1}^{\infty}$. Arranging those above sequences according to its rate of convergence then we have, $\left(\frac{1}{3 n}\right)_{n=1}^{\infty},\left(\frac{1}{4 n}\right)_{n=1}^{\infty},\left(\frac{1}{5 n}\right)_{n=1}^{\infty}, \ldots,\left(\frac{1}{(K n}\right)_{n=1}^{\infty}, \ldots$ rate of converges decreases when $\mathrm{K}$ increases. Below it is attempted to explain visually that, how does the rate of convergence decrease when $\mathrm{K}$ increases.

Here, in the below figure, each person is a representative of a particular sequence like $,\left(\frac{1}{3 n}\right)_{n=1}^{\infty},\left(\frac{1}{4 n}\right)_{n=1}^{\infty}, \ldots$ so on. We aim to show that, how does the rate of convergence of those sequences, behaves. Let's relate this to a racing game. Let each representative

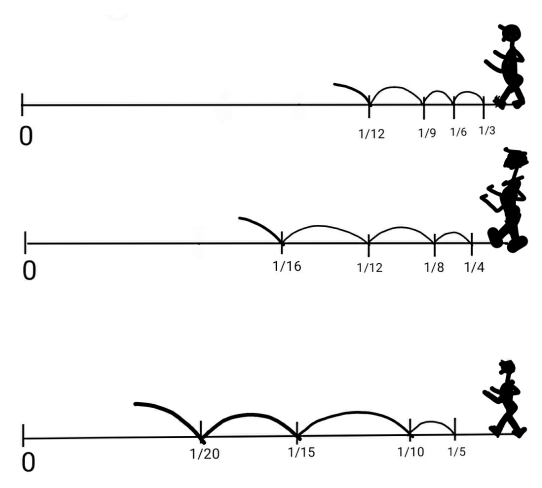

Figure 20: Visualisation to the race

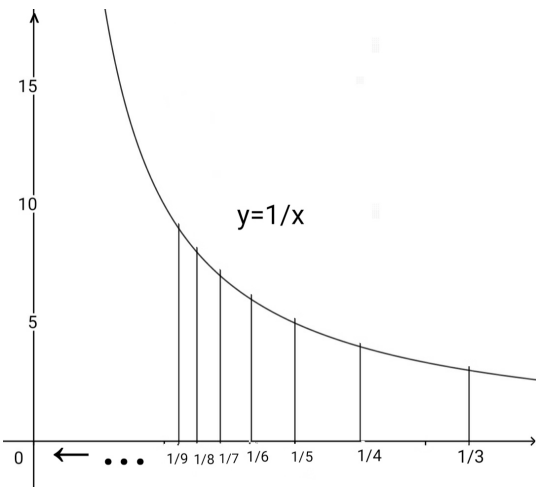

Figure 21: Decreasing order

starts its jump from its respective $1_{s t}$ sequential position and each representative takes the same time to jump one point to another, in its sequential positions. ${ }^{4}$ From the above two figures, it can be observed that the more we increase the value of $\mathrm{K}$, the better that sequence representative reaches its destination i.e. the point 0 . Now, we construct a sequence of all $T_{K}{ }^{\prime} S$ defined by,

$$
\left(T_{n}\right)_{n=1}^{\infty}=\left\{T_{1}, T_{2}, T_{3}, \ldots, T_{n}, \ldots\right\}: T_{1}>T_{2}>T_{3}>\ldots>T_{K}>\ldots
$$

So, it is decreasing in order and converging to 0 . Thus by increasing $\mathrm{n}$ in $P_{n}$, the time to get our $1_{s t}$ successor circle from the given single circle, can be reduced. Thus it can be easily observed that by increasing $\mathrm{n}$ in $P_{n}$, the time to get infinitely many successor circles from the single circle, also can be reduced.

Let, $T_{P_{K}}$ : Time to get an infinite number of circles from a single circle using $P_{K}$

\footnotetext{
${ }^{4}$ Though there is a fallacy in the above game, but don't bother about that. Just take it as a way to understand what does we mean to say.
} 
pattern ( $\mathrm{K} \geq 3$ ). So, $T_{P_{3}}>T_{P_{4}}>T_{P_{5}}>\ldots>T_{P_{K}}>\ldots$

Define, $P_{\infty}$ : the polygon with infinitely many vertices ( here, $P_{\infty}$ is just a notation ). The idea of polygonal approximation of circle tells us that the regular polygon with infinite vertices is an approximation to circle [3].

An interesting fact is that, if we apply $P_{\infty}$ pattern of the tangents on the circle then almost without taking any time, our $1_{s t}$ successor circle can be obtained and almost no time also to get our $2_{n d}$ successor circle. More precisely, if we use $P_{\infty}$ on the given single circle, then almost without taking any time, we will get infinitely many successor circles and all of those infinitely many circles merge into a single one.

Basically, the decreasing sequence, $\left(T_{P_{n}}\right)_{n=1}^{\infty}=\left\{T_{P_{1}}, T_{P_{2}}, \ldots, T_{P_{n}}, \ldots\right\}$ converges to 0 .

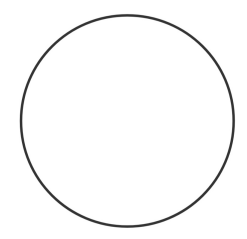

Figure 22: Merged circle

Let's deal with the thought that, how does the distance between any two nearby successive circles depends on the radius of the given single circle and also how does the radius of the successive circles vary from each other.

Let, $\mathrm{r}$ be the radius of the given single circle.

We will check for the $P_{X}(\mathrm{X} \geq 3$ ) pattern of tangents on the single circle.

Let, $b_{1}, b_{2}, b_{3}, \ldots b_{n}, \ldots$ are the end-intersecting points of tangents (for, $P_{X}$ pattern) and $u_{1}, u_{2}, u_{3}, \ldots u_{n}, \ldots$ are the circle-tangent intersecting points. The origin of the given single circle. Therefore, we will join those points with lines ( we take only those lines which passes through origin ).

Now joining points are, (i) End-intersecting point and end-intersecting point (ii) Endintersecting point and circle-tangent intersecting point (iii) Circle-tangent intersecting point and circle-tangent intersecting point.

Therefore, if we have $\mathrm{n}$ joining lines ( passing through origin ), then the whole circle is divided into $2 \mathrm{n}$ equal parts. Interestingly, for the $P_{X}$ pattern; $\mathrm{X}=\mathrm{n}$, we will have $2 \mathrm{n}$ equal parts of the circle.

At first, we will deal with the $P_{3}$ pattern (Triangular pattern) of tangents. Let, $b_{1}, b_{2}, b_{3}$ are the end-intersecting points of tangents. So, all $b_{i}{ }^{\prime} s$ lies on the circumference of the $1_{s t}$ successor circle and $u_{1}, u_{2}, u_{3}$ are the circle-tangent intersecting points. So, $o u_{1}=o u_{2}$ 

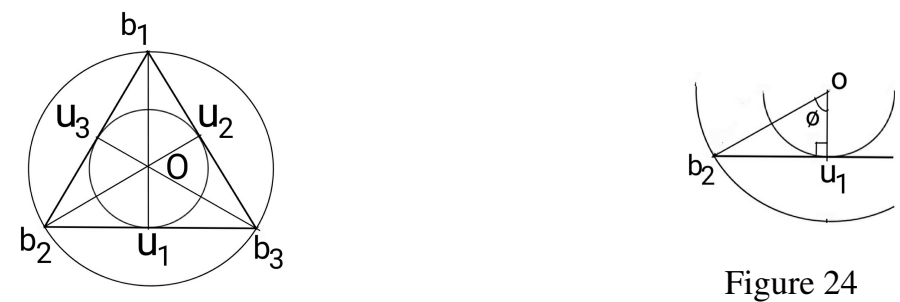

Figure 24

Figure 23: Points position

$=o u_{3}=\mathrm{r}$, i.e. the radius of the given single circle and $o b_{1}=o b_{2}=o b_{3}=r_{1}$; radius of the $1_{s t}$ successor circle. For $P_{3}$ pattern we have 3 vertices. So, previously mentioned joining lines of points would divide the whole circle into 6 equal parts. One of those equal parts is, $\triangle u_{1} o b_{2}$. It can be observed that, $o u_{1}$ is perpendicular to $b_{2} u_{1}$ and $o u_{1}=r, o b_{2}=r_{1}$. Now, $\phi=\frac{360}{6}=60$ degree ( Since, 6 equal parts ), therefore $\cos \frac{\pi}{3}=\frac{r}{r_{1}} \Rightarrow r_{1}=\frac{r}{\cos \frac{\pi}{3}}$.

Let, $r_{2}$ is the radius of the 2nd successor circle. Here, $o b_{2}=r_{2}$, $o u_{1}=r_{1}$ ( From Figure 25 ). It can be easily checked that,

$$
r_{2}=\frac{r_{1}}{\cos \frac{\pi}{3}}=\frac{r}{\left(\cos \frac{\pi}{3}\right)^{2}} .
$$

Basically, we have a sequence of radius of successor circles and it is defined below,

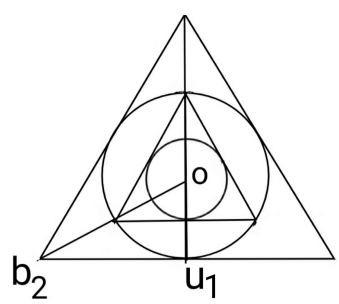

Figure 25

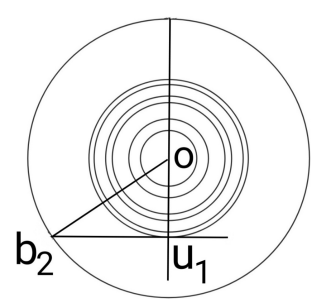

Figure 26: nth successor circle

$$
\left\{r, \frac{r}{\cos \frac{\pi}{3}}, \frac{r}{\left(\cos \frac{\pi}{3}\right)^{2}}, \ldots\right\}=\left(\frac{r}{\left(\cos \frac{\pi}{3}\right)^{j-1}}\right)_{j=1}^{\infty}
$$

Where, $\frac{r}{\left(\cos \frac{\pi}{3}\right)^{j}}$, is the radius of the $j_{t h}$ successor circle obtained using $P_{3}$ pattern. The distance between any two successive circles (including single circle also) is defined by,

$$
\left[\frac{1}{\left(\cos \frac{\pi}{3}\right)^{j}}-\frac{1}{\left(\cos \frac{\pi}{3}\right)^{j-1}}\right] r
$$


Now, we will check for $P_{4}$ pattern. Let, $b_{1}, b_{2}, b_{3}, b_{4}$ are the end-intersecting points of tangents. So, all $b_{i}{ }^{\prime} s$ lies on the circumference of the $1_{s t}$ successor circle and let, $u_{1}, u_{2}$, $u_{3}, u_{4}$ are the circle-tangent intersecting points. So, $o u_{1}=o u_{2}=o u_{3}=o u_{4}=\mathrm{r}$; radius of

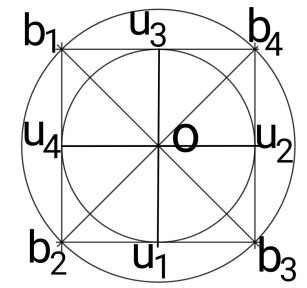

Figure 27: Points position

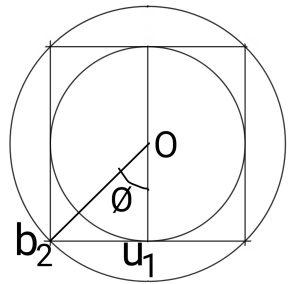

Figure 28

the given single circle and $o b_{1}=o b_{2}=o b_{3}=o b_{4}=r_{1}$; radius of the $1_{s t}$ successor circle. For $P_{4}$ pattern, the whole circle can be divided into 8 equal parts. One of those equal parts is $\triangle u_{1} o b_{2}$. It can be easily seen that, $o u_{1}$ is perpendicular to $b_{2} u_{1}$ ( here, $o u_{1}=\mathrm{r}, o b_{2}=r_{1}$ ). Now, $\phi=\frac{360}{8}=45$ degree ( Since, 8 equal parts ) therefore, $\cos \frac{\pi}{4}=\frac{r}{r_{1}} \Rightarrow r_{1}=\frac{r}{\cos \frac{\pi}{4}}$.

Let, $r_{2}$ be the radius of the $2_{n d}$ successor circle. Here, $o b_{2}=r_{2}, o u_{1}=r_{1}$. It can be easily checked that,

$$
r_{2}=\frac{r_{1}}{\cos \frac{\pi}{4}}=\frac{r}{\left(\cos \frac{\pi}{4}\right)^{2}} .
$$

For $n_{t h}$ successor circle, that one equal part will look like Figure 29. It can be easily

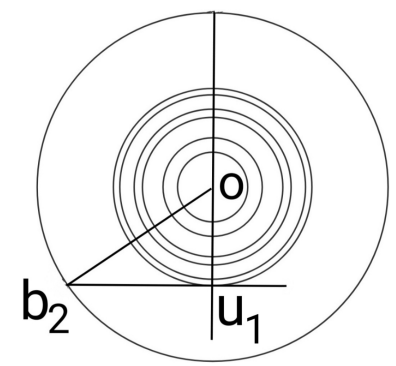

Figure 29: nth successor circle

checked that, a sequence of radius of successor circles can be obtained and each radius is depending on the radius of the given single circle. That sequence is defined below,

$$
\left\{r, \frac{r}{\cos \frac{\pi}{4}}, \frac{r}{\left(\cos \frac{\pi}{4}\right)^{2}}, \ldots\right\}=\left(\frac{r}{\left(\cos \frac{\pi}{4}\right)^{j-1}}\right)_{j=1}^{\infty}
$$


Where, $\frac{r}{\left(\cos \frac{\pi}{4}\right)^{j}}$ is the radius of the $j_{t h}$ successor circle, obtained using $P_{4}$ pattern. The distance between any two successive circles (including single circle also) is ,

$$
\left[\frac{1}{\left(\cos \frac{\pi}{4}\right)^{j}}-\frac{1}{\left(\cos \frac{\pi}{4}\right)^{j-1}}\right] r .
$$

Now, we want to propose a new theorem here.

Theorem 1. Let, we have a circle with radius $r$ and we apply $P_{X} ;(X \geq 3)$ pattern. For each $P_{X}$ pattern, we have a sequence of radius of the successor circles, depending on the radius of the given circle. Then the $(j+1)_{\text {th }}$ term of the sequence i.e. the radius of the $j_{\text {th }}$ successor circle, is defined by, $\frac{r}{\left(\cos \frac{\pi}{X}\right)^{j}}$ and the distance between any two nearby successor circles (including single circle also) is defined by,

$$
\left[\frac{1}{\left(\cos \frac{\pi}{X}\right)^{j}}-\frac{1}{\left(\cos \frac{\pi}{X}\right)^{j-1}}\right] r
$$

Now we introduce this above theorem as,

\section{" PK's Theorem for Circle ( For the polygon in which circle is circumscribed )".}

Let, the statement of the above theorem be our $\mathrm{P}(\mathrm{m})$, for some $\mathrm{m}$ belongs to $\mathrm{N}$. To prove this statement we will use 'Induction Hypothesis'.

Proof. By our previous discussions it can be easily checked that, $\mathrm{P}(1)$ and $\mathrm{P}(2)$ are true for $X=3$ and $X=4$ respectively .

Let us assume that, $\mathrm{P}(\mathrm{K})$ is true. Means, we are applying here, $P_{K+2}$ pattern of tangents - A sequence of radius of the successor circles depending on the radius of the given circle, can be obtained. Then the radius of the $j_{t h}$ successor circle is, $\frac{r}{\left(\cos \frac{\pi}{K+2}\right)^{j}}$ and the distance between any two nearby successor circles (including single circle also ) is,

$$
\left[\frac{1}{\left(\cos \frac{\pi}{K+2}\right)^{j}}-\frac{1}{\left(\cos \frac{\pi}{K+2}\right)^{j-1}}\right] r
$$

Now, we need to show that, $\mathrm{P}(\mathrm{K}+1)$ is also true.

Let, $b_{1}, b_{2}, b_{3}, \ldots, b_{K+1}$ are the end intersecting points of the tangents of the given single circle. So, all those points lie on the circumference of the $1_{s t}$ successor circle. Let, $u_{1}$ be any of the circle-tangent intersecting points and lies on the circumference of the single circle. Therefore taking lines passing through the origin, we join those points. Joining 
points are, (i). end-intersecting point and end-intersecting point (ii) end-intersecting point and circle-tangent intersecting point (iii) circle-tangent intersecting point and circletangent intersecting point.

Here we are using, $P_{K+3}$ pattern of tangents. Thus, circle can be broken into $2(\mathrm{~K}+3)$

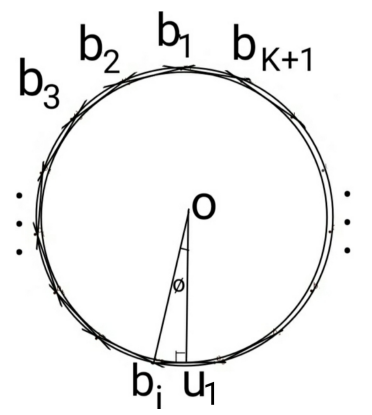

Figure 30

equal parts and each of those equal parts is a 'Right Triangle'. One of those equal part is, $\triangle o u_{1} b_{i}$, where. $o u_{1}=\mathrm{r}, o b_{i}=r_{1}$. It can be easily checked that, $\phi=\frac{360}{2(K+3)}$ degree. Therefore, $\cos \frac{\pi}{(K+3)}=\frac{r}{r_{1}} \Rightarrow r_{1}=\frac{r}{\cos \frac{\pi}{K+3}}$.

$r_{2}$ i.e. the radius of the $2_{n d}$ successor circle, can be obtained repeating the same process on the $1_{s t}$ successor circle. So now,

$$
r_{2}=\frac{r_{1}}{\cos \frac{\pi}{K+3}}=\frac{r}{\left(\cos \frac{\pi}{K+3}\right)^{2}} .
$$

Basically, we have a sequence of radius of successor circles and it is defined below,

$$
\left\{r, \frac{r}{\cos \frac{\pi}{K+3}}, \frac{r}{\left(\cos \frac{\pi}{K+3}\right)^{2}}, \ldots\right\}=\left(\frac{r}{\left(\cos \frac{\pi}{K+3}\right)^{j-1}}\right)_{j=1}^{\infty} .
$$

Here, the radius of the $j_{t h}$ successor circle is, $\frac{r}{\left(\cos \frac{\pi}{K+3}\right)^{j}}$,

and the distance between any two nearby successor circles (including given single circle also) is,

$$
\left[\frac{1}{\left(\cos \frac{\pi}{K+3}\right)^{j}}-\frac{1}{\left(\cos \frac{\pi}{K+3}\right)^{j-1}}\right] r .
$$

So, our proposed statement holds for this pattern also. Hence, $\mathrm{P}(\mathrm{K}+1)$ is true. Thus, our statement $\mathrm{P}(\mathrm{m})$ is true for all $\mathrm{m}$ belongs to $\mathrm{N}$. 


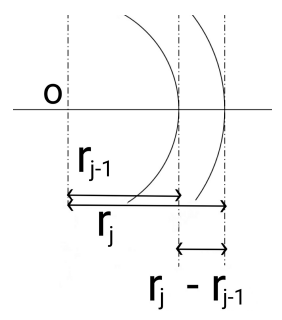

Figure 31

Hence, 'PK's Theorem for Circle ( For polygon in which circle is circumscribed )" is proved.

For the $P_{\infty}$ pattern of tangents on the single circle then we will just take the limit on the radius and distance formulae, (what we have obtained using our $P_{X} ; X \geq 3$ pattern). Therefore the radius of the $j_{t h}$ successor circle ( for $P_{\infty}$ pattern ) is,

$$
\lim _{X \rightarrow \infty} \frac{r}{\left(\cos \frac{\pi}{X}\right)^{j}}=\frac{r}{\left(\lim _{X \rightarrow \infty} \cos \frac{\pi}{X}\right)^{j}}=\frac{r}{1}=r
$$

and the distance between any two successive circles (including the single circle also) is,

$$
\lim _{X \rightarrow \infty}\left[\frac{1}{\left(\cos \frac{\pi}{X}\right)^{j}}-\frac{1}{\left(\cos \frac{\pi}{X}\right)^{j-1}}\right] r=[1-1] r=0 .
$$

So, it can be observed that, for $P_{\infty}$ pattern of tangents, all the infinitely many successor circles has almost the same radius, as what the single circle has, i.e. all the infinitely many successor circles merge into a single one, almost ${ }^{5}$ without taking any time.

Interestingly, if our given circle has zero radius then whatever $P_{X}$ pattern we apply on it, it will always remain a single point.

\subsection{PREDECESSOR CIRCLES.}

In this portion, it is attempted to discuss that, how do we can reach up into a single point from a given circle using polygonal approximation.

Let us assume that, each circle is made up of a polygonal approximation. From the discussions of the previous portion of this article, it follows that we always have a predecessor circle for each circle ( It can be easily observed! ). The below algorithm describes the whole process in a precise way.

\footnotetext{
${ }^{5}$ We mentioned 'almost', because we use polygonal approximation to get such observation and also in Limit, we are not interested in the arrival, rather we are interested in approaching .
} 


\subsubsection{ALGORITHM.}

Step 1 : Take a single circle.

Step 2: Assume that, each circle is made up of a polygonal approximation. Therefore each circle will always give a predecessor circle.

Step 3: For any $P_{X}(X \geq 3)$ polygon pattern, applying the previous assumption (Step 2 ), our $1_{s t}$ predecessor circle can be obtained.

Step 4: Repeat the same process on $1_{s t}$ predecessor circle to get the $2 n d$ predecessor circle.

Step 5: Repeating the same procedure again and again, one can obtain more and more predecessor circles.

Step 6: The more we repeat this process again and again, the better we can observe that the radius of the predecessor circles becomes smaller and smaller.

Step 7: Applying this process infinitely many times, then a single point is almost reached! ( We mentioned 'almost' here, since we are using approximations to tell so ).

Step 8: If one reached up just into a single point ( instead of almost into a single point ) then there would arise a fallacy ${ }^{6}$. That's why it is better to use the word, 'almost'.

\subsubsection{TIME.}

The below picture gives us an idea of polygonal approximation of circle.

It can be observed that area of any n-regular polygon inscribed in a circle has a smaller

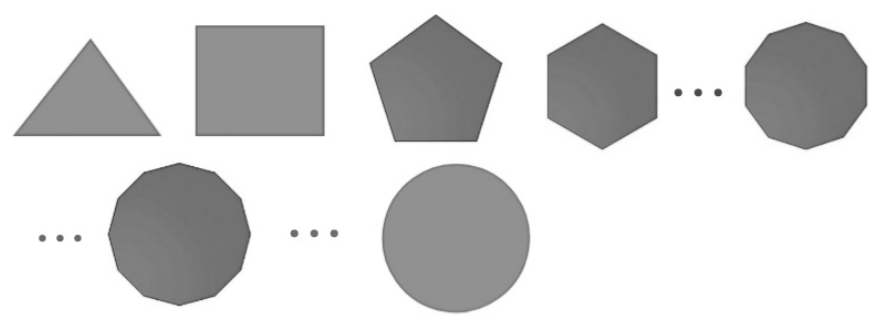

Figure 32: Polygonal Approximation

( or, almost the same ) area than the area of the circle. The more the value of $\mathrm{X}$ in $P_{X}$ increases, the better it can be observed that the area of the regular polygon also increases. For $P_{\infty}$ pattern, the area of the regular polygon becomes almost the same as the area of the given circle [3]. i.e. area of inscribed triangle $<$ area of inscribed square $<$ area of inscribed pentagon $<\ldots \leq$ area of the circle . Let us consider that,

$T_{1}$ be the time to reach up into a single point from the given single circle, using $P_{3}$ pattern.

\footnotetext{
${ }^{6}$ The fallacy is that by applying reverse process we can reach back into our given circle again from the single point. But, if it's just a point then its radius is zero. For that case, whatever polygonal pattern of tangents we apply to it, it will always remain a single point. This means we will never get our given circle
} 
$T_{2}$ be the required time for using $P_{4}$ pattern.

...

$T_{K}$ be the required time for using $P_{K+2}$ pattern.

...

$T_{\infty}$ be the required time for using $P_{\infty}$ pattern. ( Here, $T_{\infty}$ and $P_{\infty}$ are just a notation )

Claim: The sequence $\left(T_{n}\right)_{n=1}^{\infty}$ is increasing in order i.e. $T_{1}<T_{2}<T_{3}<\ldots<T_{n}<$ $\ldots$ and it is a divergent sequence.

To Establish : $r_{j}$ for $P_{3}<r_{j}$ for $P_{4}<\ldots<r_{j}$ for $P_{k}<\ldots<r_{j}$ for $P_{n}<\ldots$ and $r_{j}$ for $P_{X} \geq r_{j+1}$ for $P_{X} \geq r_{j+2}$ for $P_{X} \geq \ldots$. For $P_{\infty}$ pattern, $\lim _{X \rightarrow \infty} r_{j}=r$ and $\lim _{X \rightarrow \infty}\left(r_{j-1}-r_{j}\right)=0$, for all $\mathrm{j}$ belongs to $\mathrm{N}$.

Proof. Let, $A_{1}, A_{2}, A_{3}$ are the vertices of any inscribed triangle (i.e. $P_{3}$ ) and $V_{1}$, $V_{2}, V_{3}$ are the $1_{s t}$ predecessor circle and polygon $P_{3}$ intersecting points. Since here we

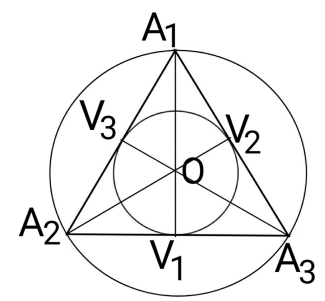

Figure 33

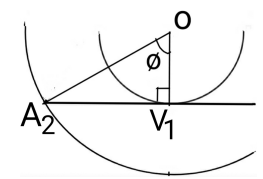

Figure 34

are dealing with the $P_{3}$ pattern, we can divide the given circle into 6 equal parts. Each of them is a right triangle. One of those equal parts is, $\triangle \mathrm{A}_{2} \mathrm{O} V_{1}$. Now, $\phi=\frac{360}{6}=60$ degree ( Since, 6 equal parts ).

Let, $\mathrm{O} A_{2}=\mathrm{r}$ : radius of the given single circle and $\mathrm{O} V_{1}=r_{1}$ : radius of the $1_{s t}$ predecessor circle. Therefore, $\cos \frac{\pi}{3}=\frac{r_{1}}{r} \Rightarrow r_{1}=\left(\cos \frac{\pi}{3}\right) r$.

Let, $r_{2}$ be the radius of the $2_{n d}$ predecessor circle. It can be easily checked that,

$$
r_{2}=\left(\cos \frac{\pi}{3}\right) r_{1} \Rightarrow r_{2}=\left(\cos \frac{\pi}{3}\right)^{2} r .
$$

Continuing like this, a sequence of the radius of predecessor circles can be obtained.

$$
\left\{r,\left(\cos \frac{\pi}{3}\right) r,\left(\cos \frac{\pi}{3}\right)^{2} r, \ldots\right\}=\left(\left(\cos \frac{\pi}{3}\right)^{j-1} r\right)_{j=1}^{\infty} .
$$

The $j_{t h}$ term of the above sequence i.e. $\left(\cos \frac{\pi}{3}\right)^{j} r$, is the radius of the $j_{t h}$ predecessor circle. It can be observed that, the distance between any two predecessor circles (including 
given single circle also) is defined by,

$$
\left[\left(\cos \frac{\pi}{3}\right)^{j-1}-\left(\cos \frac{\pi}{3}\right)^{j}\right] r
$$

Let's check for $P_{4}$ pattern. Let, $A_{1}, A_{2}, A_{3}, A_{4}$ are the vertices of any inscribed square ( i.e. $P_{4}$ ) and $V_{1}, V_{2}, V_{3}, V_{4}$ are the $1_{s t}$ predecessor circle and polygon $P_{4}$ intersecting points. Since here we are dealing with $P_{4}$ pattern, given circle can be divided into 8 equal parts. Each of them is a right triangle. One of those equal parts is, $\triangle \mathrm{A}_{2} \mathrm{O} V_{1}$. Now, $\phi=\frac{360}{8}=45$ degree ( Since, 8 equal parts ). Let, $\mathrm{O} A_{2}=\mathrm{r}$ is the radius of the
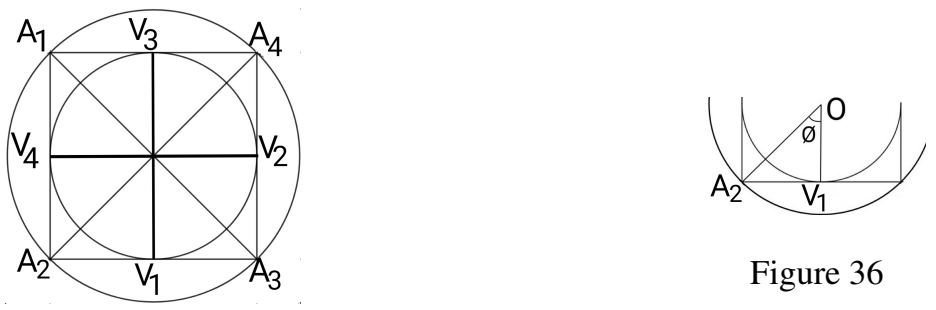

Figure 36

Figure 35

given single circle and $\mathrm{O} V_{1}=r_{1}$ is the radius of the $1_{s t}$ predecessor circle. Therefore, $\cos \frac{\pi}{4}=\frac{r_{1}}{r} \Rightarrow r_{1}=\left(\cos \frac{\pi}{4}\right) r$.

Let, $r_{2}$ be the radius of the 2 nd predecessor circle. It can be easily checked that,

$$
r_{2}=\left(\cos \frac{\pi}{4}\right) r_{1} \Rightarrow r_{2}=\left(\cos \frac{\pi}{4}\right)^{2} r
$$

Interestingly, we have a sequence of radius ,

$$
\left(\left(\cos \frac{\pi}{4}\right)^{j-1} r\right)_{j=1}^{\infty}=\left\{r,\left(\cos \frac{\pi}{4}\right) r,\left(\cos \frac{\pi}{4}\right)^{2} r, \ldots\right\}
$$

It can be easily observed that, $\left(\cos \frac{\pi}{4}\right)^{j} r$ is the radius of the $j_{t h}$ predecessor circle and the distance between any two predecessor circle (including given single circle also) is,

$$
\left[\left(\cos \frac{\pi}{4}\right)^{j-1}-\left(\cos \frac{\pi}{4}\right)^{j}\right] r
$$

Now here, we wants to propose a new theorem.

Theorem 2. Let, we have a circle with radius $r$ and we apply $P_{X} ;(X \geq 3)$ pattern. For each $P_{X}$ pattern, we have a sequence of radius of the predecessor circles, depending on the radius of the given circle. Then the $(j+1)_{\text {th }}$ term of the sequence i.e. the radius of 
the $j_{\text {th }}$ predecessor circle is defined by, $\left(\cos \frac{\pi}{X}\right)^{j} r$ and the distance between any two nearby predecessor circle (including given single circle also) is defined by,

$$
\left[\left(\cos \frac{\pi}{X}\right)^{j-1}-\left(\cos \frac{\pi}{X}\right)^{j}\right] r
$$

Now we introduce this above theorem as,

\section{"PK's Theorem for Circle ( For inscribed polygon )".}

Let, the statement of the above theorem be our $\mathrm{P}(\mathrm{m})$, for some $\mathrm{m}$ belongs to $\mathrm{N}$. To prove this statement we will use 'Induction Hypothesis'.

Proof. It can be easily checked that $\mathrm{P}(1)$ and $\mathrm{P}(2)$ are true for $\mathrm{X}=3$ and $\mathrm{X}=4$ respectively. Let us assume that, $\mathrm{P}(\mathrm{K})$ is true. Means, we are applying here, $P_{K+2}$ pattern . A sequence of radius of the predecessor circles depending on the radius of the given circle, can be obtained. Then the radius of the $j_{t h}$ predecessor circle is, $\left(\cos \frac{\pi}{K+2}\right)^{j} r$ and the distance between any two nearby predecessor circles (including single circle also) is,

$$
\left[\left(\cos \frac{\pi}{K+2}\right)^{j-1}-\left(\cos \frac{\pi}{K+2}\right)^{j}\right] r
$$

Now, we need to show that, $\mathrm{P}(\mathrm{K}+1)$ is also true.

Let, $A_{1}, A_{2} \ldots A_{i} \ldots$ are the vertices of the inscribed polygon ( $P_{K+2}$ ) of a circle with radius $\mathrm{r}$. Each mid point of the side $A_{i} A_{i+1}$ : for all $\mathrm{i}$ belongs to $\mathrm{N}$, is a polygonpredecessor circle intersecting point. Let, $V_{1}$ be one of those polygon- predecessor circle intersecting point. Here in the above figure, $O V_{1}=r_{1}$ : radius of the $1_{\text {st }}$ predecessor

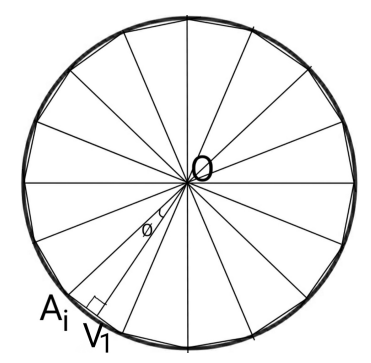

Figure 37

circle and $O A_{i}=\mathrm{r}$ : radius of the given single circle. The whole given circle can be broken into $2(\mathrm{~K}+3)$ equal parts. One of those equal parts is $\triangle A_{i} O V_{1}$. Let, $\phi$ be the angle between $O V_{1}$ and $O A_{i}$. Now, $\phi=\frac{360}{2(K+3)}$ degree ( Since, 2(K+3) equal parts ). Therefore $\cos \frac{\pi}{K+3}=\frac{r_{1}}{r} \Rightarrow r_{1}=\left(\cos \frac{\pi}{K+3}\right) r$. 
Repeating the same process on the $1_{s t}$ predecessor circle, $r_{2}$ i.e. the radius of the $2_{n d}$ predecessor circle, can be obtained. So now,

$$
r_{2}=\left(\cos \frac{\pi}{K+3}\right) r_{1} \Rightarrow r_{2}=\left(\cos \frac{\pi}{K+3}\right)^{2} r
$$

Interestingly, applying this process again and again we will have a sequence of radius

$$
\left(\left(\cos \frac{\pi}{K+3}\right)^{j-1} r\right)_{j=1}^{\infty}=\left\{r,\left(\cos \frac{\pi}{K+3}\right) r,\left(\cos \frac{\pi}{K+3}\right)^{2} r, \ldots\right\} .
$$

Here, the radius of the $j_{t h}$ predecessor circle is $\left(\cos \frac{\pi}{K+3}\right)^{j} r$, and the distance between any two nearby predecessor circles (including single circle also) is,

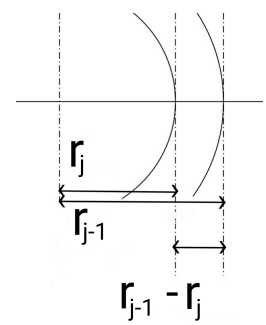

Figure 38

$$
\left[\left(\cos \frac{\pi}{K+3}\right)^{j-1}-\left(\cos \frac{\pi}{K+3}\right)^{j}\right] r
$$

So, $\mathrm{P}(\mathrm{K}+1)$ is true. Thus, our statement $\mathrm{P}(\mathrm{m})$ is true for all $\mathrm{m}$ belongs to $\mathrm{N}$.

Hence, “ PK's Theorem for Circle ( For inscribed polygon )" is proved.

For the $P_{\infty}$ pattern of tangents on the single circle then we will just take the limit on the radius and distance formulae, (what we have obtained using our $P_{X} ; X \geq 3$ pattern ). Therefore the radius of the $j_{t h}$ successor circle ( for $P_{\infty}$ pattern ) is, $\lim _{X \rightarrow \infty}\left(\cos \frac{\pi}{X}\right)^{j} r=$ $r$, and the distance between any two nearby predecessor circles (including the single circle also) is,

$$
\lim _{X \rightarrow \infty}\left[\left(\cos \frac{\pi}{X}\right)^{j-1}-\left(\cos \frac{\pi}{X}\right)^{j}\right] r=[1-1] r=0 .
$$

Basically, it can be observed that, $r_{j} \rightarrow r$ and $\left(r_{j-1}-r_{j}\right) \rightarrow 0$ when $X \rightarrow \infty$. We need to compare, $r_{1}$ for $P_{3}=r \cos \frac{\pi}{3}, r_{1}$ for $P_{4}=r \cos \frac{\pi}{4}, \ldots, r_{1}$ for $P_{X}=r \cos \frac{\pi}{X} \ldots$ 
Since, $r>0$. So enough to compare the cosine parts. Here, $\lim _{X \rightarrow \infty} \cos \frac{\pi}{X}=1$ and

$$
\begin{array}{r}
\cos \frac{\pi}{3}<\cos \frac{\pi}{4}<\cos \frac{\pi}{5}<\ldots<\cos \frac{\pi}{X}<\ldots \\
\Rightarrow\left(\cos \frac{\pi}{3}\right)^{2}<\left(\cos \frac{\pi}{4}\right)^{2}<\left(\cos \frac{\pi}{5}\right)^{2}<\ldots<\left(\cos \frac{\pi}{X}\right)^{2}<\ldots \\
\ldots \\
\Rightarrow\left(\cos \frac{\pi}{3}\right)^{j}<\left(\cos \frac{\pi}{4}\right)^{j}<\left(\cos \frac{\pi}{5}\right)^{j}<\ldots<\left(\cos \frac{\pi}{X}\right)^{j}<\ldots
\end{array}
$$

For all $\mathrm{j}$ belongs to $\mathrm{N}$.

From equation (1) we have, $r_{1}$ for $P_{3}<r_{1}$ for $P_{4}<r_{1}$ for $P_{5}<\ldots r_{1}$ for $P_{X}<\ldots$ and from equation (2) we have, $r_{2}$ for $P_{3}<r_{2}$ for $P_{4}<r_{2}$ for $P_{5}<\ldots r_{2}$ for $P_{X}<\ldots$ and from equation (3) we have, $r_{j}$ for $P_{3}<r_{j}$ for $P_{4}<r_{j}$ for $P_{5}<\ldots r_{j}$ for $P_{X}<\ldots$ Therefore, $r_{j}$ for $P_{X} \geq r_{j+1}$ for $P_{X} \geq r_{j+2}$ for $P_{X} \geq \ldots$ since, $\cos x$ is bounded above by 1 . Thus, $\cos x \geq(\cos x)^{2} \geq(\cos x)^{3} \geq \ldots$.

It can be conclude that, $T_{1}<T_{2}<T_{3}<\ldots$ i.e. $\left(T_{n}\right)_{n=1}^{\infty}$ is increasing. For $P_{\infty}$ pattern, each $r_{i}$ is almost equals to $\mathrm{r}$. Thus it takes infinite time to reach up, from a given circle into a single point, i.e. the circle with zero radius . Meaning, for $P_{\infty}$ pattern, $T_{\infty}$ is infinite.Thus, $\left(T_{n}\right)_{n=1}^{\infty}$ is not bounded above. More precisely, $\left(T_{n}\right)_{n=1}^{\infty}$ is divergent.

Hence, our claim is proved.

\section{CONCLUDING REMARKS.}

In this paper, we described how does one can obtain infinitely many circles from a single circle, using its tangents with a pattern, and how a single point can be obtained from a given circle. Later we mentioned the algorithms behind it. We also attempted to give an idea about how does time behaves for those observations. Furthermore, for the $P_{\infty}$ pattern all the infinite successor circles merge into a single one, almost without taking any time and it takes infinite time to reach up into a single point. In this whole article, we introduced two new theorems for inscribed polygon and for the polygon in which circle is circumscribed.

Acknowledgement: The author would like to express his gratitude to his teachers Dr. Sougata Mitra, Dr. Sajith G. , Dr. Sabyasachi Bhattacharya, and TeXpert Ahmed A. Aafaq for their valuable comments and suggestions and his parents for their continuous support. 


\section{References}

[1] Claudi Alsina and Roger B. Nelsen, Charming Proofs: A Journey into Elegant Mathematics, Dolkani Mathematical Expositions Series, 2010.

[2] Terrance Tao, Analysis I and Analysis II, Texts and Readings in Mathematics ( TRIM vol - 37 and $\mathbf{3 8}$ ), Springer.

[3] Muharrem Aktumen and Ahmet Kacar, Maplets for the Area of the Unit Circle , The Journal of Online Mathematics and Its Applications, Vol-7 , May (2007), Mathematical Association of America, https://www.maa.org/external_ archive/joma/Volume7/Aktumen/index.html.

[4] A Brief History of Pi ( $\pi$ ), Exploratorium, https://www.exploratorium. edu/pi/history-of-pi.

[5] L Yong-Kui, Algorithm for circle approximation and generation, Computer-Aided Design, Vol-25 , No.3 (1993), PP. 169-171, https://doi.org/10.1016/ $0010-4485$ (93) 90042-M.

[6] Józef Borkowski, Minimization of Maximum Errors In Universal Approximation of The Unit Circle By a Polygon , Metrol. Mess. Syst. , vol- XVIII (2011), no.3 , pp 391-402.

[7] J. M. Carnicer, E. Mainar and J. M. Peña (2019), A totally positive basis for circle approximations, Revista de la Real Academia de Ciencias Exactas, Físicas y Naturales. Series A. Matemáticas, 113 , PP 3383-3397; https : / / doi .org/ 10 . $1007 /$ s13398-019-00705-5.

PALASH KHANRA ๑

DEPT. OF MATHEMATICS

RAMAKRISHNA MISSION VIDYAMANDIRA

BELUR MATH, PIN-711202, INDIA

E-mail: palash1729@vidyamandira.ac.in 